\title{
Inventory Replenishment Policy with Time and Reliability Varying Demand
}

\author{
N.P. Behera ${ }^{1^{*}}$, P. K. Tripathy ${ }^{2}$ \\ ${ }^{1 *}$ Department of Statistics, Utkal University, Bhubaneswar, INDIA \\ ${ }^{2}$ Department of Statistics, Utkal University, Bhubaneswar, INDIA \\ *Corresponding Author: nalinipbehera@gmail.com,Tel: 9439957202
}

Available online at: www.isroset.org

Received 07/Mar/2018, Revised 15/Mar/2018, Accepted 10/Apr/2018, Online 30/Apr/2018

\begin{abstract}
In real life situation, demand may be increase, decrease or constant. But, in our present study demand is assumed to be an increasing function of time which depends on reliability. Shortages are allowed and excess demand is backlogged. The economic production lot size and the reliability of the production process along with the production period are the decision variables and total cost per cycle is the objective function which is to be minimized. Further the parameters involved in the business may likely to be changed due to the fast growing marketing system. Therefore; it will be more realistic and market friendly to deal with a fuzzy model rather than a crisp model. Both crisp and fuzzy models have been proposed to determine the optimal solution. The demand, shortage cost, holding cost and deterioration rate and reliability are considered as pentagonal fuzzy numbers. Defuzzification of the total cost has been carried out by Graded Mean Representation Method and Signed Distance Method. Sensitivity analysis is also incorporated to investigate the effect of different system parameters in enhancing the cost.
\end{abstract}

Keywords: Reliability, Pentagonal Fuzzy Number, Graded Mean Representation Method, Signed Distance Method.

Mathematics Subject Classification: 90B05

\section{INTRODUCTION}

Inventory control is the supervision of supply, storage and accessibility of items in order to ensure an adequate supply without excessive oversupply. It can also be referred as the process of managing the timing and the quantities of goods to be ordered and stocked, so that demands can be met satisfactorily and economically. Success of inventory control depends on some important issues, i.e., uncertainty about the size of future demands, uncertainty of inventory cost, uncertainty of deterioration, reliability of the production process, etc. In the above cases, it has been assumed that the inventory parameters are crisp or precise or probabilistic but in reality they may deviate a little from their actual value without following any probability distribution. To deal with such type of uncertainty in inventory parameters, the notion of fuzziness has been initialized by Zimmermann [4]. This model has also been maneuvered by various researchers. Tripathy et al. established a fuzzy Economic Order Quantity model with reliability where the unit cost depends on demand [3]. Dutta and Kumar developed a fuzzy inventory model without shortage where holding cost and ordering cost were taken as trapezoidal fuzzy numbers [1]. Tripathy and Pattnaik focused on optimal disposal mechanism by considering the system cost as fuzzy under flexibility and reliability criteria [5]. Tripathy and Behera formulated a fuzzy inventory model for time deteriorating items using penalty cost under the condition of infinite production rate [6]. Dutta and Kumar explored an optimal ordering policy for an inventory model for deteriorating items without shortage where demand rate, ordering cost and holding cost were taken as fuzzy in nature [2]. Jaggi et al. introduced a fuzzy inventory model for deteriorating items with time varying demand and shortages [8]. Singh and Rathore studied an inventory model for deteriorating items with reliability consideration and trade credit [7].

The present study elaborates the above model in fuzzy environment and compares the result obtained in crisp and fuzzy environment to prove the superiority of the proposed model over the crisp model in achieving the optimal 
solution. Here shortage cost, Holding cost, deterioration rate and reliability are considered as pentagonal fuzzy numbers. Defuzzification of the total cost has been accomplished by Graded Mean Representation Method and Signed Distance Method. Numerical examples have been cited as a proof of the validation of the model. Sensitivity analysis has also been carried out to prove the credibility of the proposed model over crisp model.

The remainder of this paper is organized as follows. In the next section, the definition and preliminaries are described. Section III presents the assumptions and notations. Section IV describes the mathematical model and solution procedure. In section $\mathrm{V}$ numerical examples are presented to illustrate the model and the sensitivity analysis of the optimal solution with respect to parameter of the system is also carried out. Section VI contains the results and discussion. Section VII presents the conclusion with future directions.

\section{DEFINITIONS AND PRELIMINARIES}

We first recapitulate some underlying definitions and basic results of fuzzy numbers.

Fuzzy Set: Fuzzy set is a vague boundary set as compared to the crisp set. It is a collection of objects in connection with expression of uncertainty of the property characterizing the objects by grades from the interval between 0 and 1 .

Membership function: In fuzzy set each element is mapped to $[0,1]$ by membership function.

$$
\mu_{A}: X \rightarrow[0,1]
$$

$\propto$-Cut set: The $\propto$-cut of a fuzzy set $A_{\propto}$ is made up of members whose membership function is not less than $\propto$.

Fuzzy number: Fuzzy number is expressed as a fuzzy set defining a fuzzy interval in the real number $R$. Generally a fuzzy interval is expressed by two end points and a peak point. The $\propto$-cut operation can also be applied to the fuzzy number. If we denote $\propto$-cut interval for fuzzy number $A$ as $A_{\propto}$, the obtained interval $A_{\propto}$ is defined as

$$
A_{\alpha}=\left\lfloor a_{1}^{\alpha}, a_{3}^{\alpha}\right\rfloor
$$

(PFN):

\section{Pentagonal fuzzy number}

$$
\begin{gathered}
C_{R_{1}}(\alpha)=d-(d-c) \alpha=R_{1}^{-1}(\alpha) \\
C_{R_{2}}(\alpha)=e-(e-d) \alpha=R_{2}^{-1}(\alpha)
\end{gathered}
$$

Due to error in measuring technique, instrumental faultiness, etc some data in our observation cannot be precisely or accurately determined. Let us consider that we measure the weather temperature and humidity simultaneously. The temperature is approximately $35^{\circ} \mathrm{C}$ with normal humidity i.e. the temperature is not perfect either more or less than $35^{\circ} \mathrm{C}$, which affects normal humidity in the atmosphere. Thus, variation in temperature also affects the percentages of humidity. This phenomenon happens in general. This concept of variation leads to a new type of fuzzy number called the pentagonal fuzzy number is a 5-tuple subset of a real number having 5 parameters.

A pentagonal fuzzy number $C=\left(c_{1}, c_{2}, c_{3}, c_{4}, c_{5}\right)$, where $c_{3}$ is the middle point and $\left(c_{1}, c_{2}\right)$ and $\left(c_{4}, c_{5}\right)$ are the left and right side points of $c_{3}$, respectively. Now, we consider the mathematical definition of a pentagonal fuzzy number.

Definition 1: Pentagonal fuzzy number: A fuzzy number $C=\left(c_{1}, c_{2}, c_{3}, c_{4}, c_{5}\right)$ is called a pentagonal fuzzy number when the membership function has the form

$$
\mu_{C}\left(x ; w_{1}, w_{2}\right)=\left\{\begin{array}{lc}
\mathrm{L}_{1}(x)=\frac{x-c_{1}}{c_{2}-c_{1}}, & c_{1} \leq x \leq c_{2} \\
L_{2}(x)=\frac{x-c_{2}}{c_{3}-c_{2}}, & c_{2} \leq x \leq c_{3} \\
1 & x=c_{3} \\
R_{1}(x)=\frac{x-c_{3}}{c_{4}-c_{3}}, & c_{3} \leq x \leq c_{4} \\
R_{2}(x)=\frac{x-c_{5}}{c_{4}-c_{5}}, & c_{4} \leq x \leq c_{5} \\
0, & \mathrm{x}>c_{5}
\end{array}\right.
$$

The $\quad \alpha$-cut of $C=\left(c_{1}, c_{2}, c_{3}, c_{4}, c_{5}\right), \quad 0 \leq \alpha \leq 1 \quad$ is $(\alpha)=\left[C_{L}(\alpha), C_{R}(\alpha)\right]$

Where, $C_{L_{1}}(\alpha)=a+(b-a) \alpha=L_{1}^{-1}(\alpha)$

$C_{L_{2}}(\alpha)=b+(c-d) \alpha=L_{2}^{-1}(\alpha)$

$$
\begin{aligned}
& \text { So, } \quad L^{-1}(\alpha)=\frac{1}{2}\left[{L_{1}}^{-1}(\alpha)+L_{2}{ }^{-1}(\alpha)=\frac{1}{2}[a+b+(c-a) \alpha]\right] \\
& R^{-1}(\alpha)=\frac{1}{2}\left[R_{1}^{-1}(\alpha)+R_{2}^{-1}(\alpha)\right]=\frac{1}{2}[d+e-(e-c) \alpha]
\end{aligned}
$$

Definition 2: If $C=\left(c_{1}, c_{2}, c_{3}, c_{4}, c_{5}\right)$ is a pentagonal fuzzy number then the graded mean representation method 
of $\tilde{C}$ is defined a $P(\tilde{C})=\frac{\int_{0}^{w} C\left(\frac{L^{-1}(h)+R^{-1}(h)}{2}\right) d h}{\int_{0}^{w_{c}} h d h}$,

$0 \leq h \leq w_{C}$ and $0 \leq w_{C} \leq 1$

$P(\tilde{C})=\frac{c_{1}+3 c_{2}+4 c_{3}+3 c_{4}+c_{5}}{12}$

Definition 3: Let $C=\left(c_{1}, c_{2}, c_{3}, c_{4}, c_{5}\right)$ is a pentagonal fuzzy number then the signed distance of $\tilde{C}$ is defined as $d(C, 0)=\int_{0}^{1} d\left(\left[C_{L}(\alpha), C_{R}(\alpha)\right], 0\right)=\frac{1}{8}\left(c_{1}+2 c_{2}+2 c_{3}+2 c_{4}+c_{5}\right)$. Arithmetic Operations of PFN:

Formation of an arithmetic operation is crucial in the study of fuzzy numbers; the author tries to establish some basic arithmetic operations of PFN.

(1) Addition: Let $C=\left(c_{1}, c_{2}, c_{3}, c_{4}, c_{5}\right)$ and

$D=\left(d_{1}, d_{2}, d_{3}, d_{4}, d_{5}\right)$ be two PFNs: then,

$C+D=\left(c_{1}+d_{1}, c_{2}+d_{2}, c_{3}+d_{3}, c_{4}+d_{4}, c_{5}+d_{5}\right)$.

(2) Subtraction: we define the subtraction of two

PFNs $C=\left(c_{1}, c_{2}, c_{3}, c_{4}, c_{5}\right)$ and

$D=\left(d_{1}, d_{2}, d_{3}, d_{4}, d_{5}\right)$, we get

$C-D=\left(c_{1}-d_{1}, c_{2}-d_{2}, c_{3}-d_{3}, c_{4}-d_{4}, c_{5}-d_{5}\right)$.

Scalar Multiplication: Let $C=\left(c_{1}, c_{2}, c_{3}, c_{4}, c_{5}\right)$ be a PFN

and $k \in R$ be any scalar. If

$k \geq 0, \mathrm{kC}=\left(k c_{1}, k c_{2}, k c_{3}, k c_{4}, k c_{5}\right)$.

(4) Multiplication: Let $C=\left(c_{1}, c_{2}, c_{3}, c_{4}, c_{5}\right)$ and $D=\left(d_{1}, d_{2}, d_{3}, d_{4}, d_{5}\right)$ be two PFNs: then,

$C * D=\left(c_{1} * d_{1}, c_{2} * d_{2}, c_{3} * d_{3}, c_{4} * d_{4}, c_{5} * d_{5}\right)$.

(5) Inverse: we define the inverse of a PFN when all its components are non zero. Suppose $C=\left(c_{1}, c_{2}, c_{3}, c_{4}, c_{5}\right)$ is a PFN: then,

$C^{-1} \approx \frac{1}{C} \approx\left(\frac{1}{c_{5}}, \frac{1}{c_{4}}, \frac{1}{c_{3}}, \frac{1}{c_{2}}, \frac{1}{c_{1}}\right)$
If one of the components of a PFN becomes zero; then we cannot find its inverse.

(6) Division: the division of two PFNs $C=\left(c_{1}, c_{2}, c_{3}, c_{4}, c_{5}\right)$ and $D=\left(d_{1}, d_{2}, d_{3}, d_{4}, d_{5}\right) \quad$ is approximated as the multiplication with inverse.

$$
\frac{C}{D} \approx C D^{-1} \approx\left(\frac{c_{1}}{d_{5}}, \frac{c_{2}}{d_{4}}, \frac{c_{3}}{d_{3}}, \frac{c_{4}}{d_{2}}, \frac{c_{5}}{d_{1}}\right)
$$

(7) Exponent: The exponent of a PFN $C=\left(c_{1}, c_{2}, c_{3}, c_{4}, c_{5}\right)$ is defined as the power of its components. $\quad C^{n} \approx\left(c_{1}{ }^{n}, c_{2}{ }^{n}, c_{3}{ }^{n}, c_{4}{ }^{n}, c_{5}{ }^{n}\right)$

\section{ASSUMPTIONS AND NOTATIONS}

We adopt the following assumptions and notations for the models to be discussed.

\section{Assumptions:}

(i) Demand $R(t)=a(1+r)^{t}$ is assumed to be an increasing function of time and reliability i.e $a$ is the positive constant and $a>0$.

(ii) Replenishment rate is infinite.

(iii) Lead time is zero.

(iv) Shortages are allowed and fully backlogged.

\section{Notations:}

(i) $R(t)$ : Demand rate for any time per unit time. (ii) $A_{0}$ : Ordering cost per order.

(iii) $\theta_{0}:$ Deterioration rate.

(iv) $\mathrm{T}$ : Length of the cycle.

(v) $Q_{0}$ : Ordering quantity per unit time.

(vi) $h_{0}$ : Holding cost.

(vii) $S_{0}:$ Shortages cost.

(viii) $C_{0}$ : Unit cost per unit time.

(ix) $r$ : Reliability of the product.

(x) $\phi\left(t_{1}, T\right)$ : Total inventory cost per unit time.

(xi) $\tilde{R}$ : Fuzzy demand. 
(xii) $\tilde{\theta}_{0}$ : Fuzzy deterioration rate.

(xiii) $\tilde{h}_{0}$ : Fuzzy holding cost per unit time.

(xiv) $\tilde{S}_{0}:$ Fuzzy shortages cost per unit time.

(xv) $\tilde{C}_{0}$ : Fuzzy unit cost per unit time.

(xvi) $\widetilde{\phi}\left(t_{1}, T\right)$ : Total fuzzy inventory cost per unit time.

(xvii) $\tilde{\phi}_{G M}\left(t_{1}, T\right)$ : Defuzzified value of $\tilde{\phi}\left(t_{1}, T\right)$ by applying Graded mean representation method.

(xviii) $\tilde{\phi}_{S D}\left(t_{1}, T\right)$ : Defuzzified value of $\tilde{\phi}\left(t_{1}, T\right)$ by applying Signed distance method.

(xix) $t_{1}$ : Optimal time period of the positive stock.

\section{MATHEMATICAL MODEL}

Let $Q_{1}$ be the inventory level at the beginning of the cycle. Amid the period $\left(0, \mathrm{t}_{1}\right)$, the depletion in the inventory occurs due to demand and deterioration. Ultimately, inventory reaches zero level at time $\mathrm{t}_{1}$. The period $\left(\mathrm{t}_{1}, T\right)$ is the period of shortages, which are fully backlogged. Then the behavior of the inventory level is governed by the following differential equations.

\section{Crisp Model:}

$$
\frac{d q(t)}{d t}+\theta_{0} q(t)=-R(t) \quad 0 \leq t \leq t_{1}
$$

Deterioration $\operatorname{cost}\left(q_{d c}\right)$ in the cycle is given by

$q_{d c}=Q_{0}-\int_{0}^{t_{1}} q(t) d t=a\left[\frac{\theta_{0} t_{1}{ }^{2}}{2}+\frac{\theta_{0} r t_{1}{ }^{3}}{3}\right]$

Shortage cost $\left(q_{s c}\right)$ in the cycle is given by

$q_{s c}=-\left[a\left(t_{1} T-\frac{t_{1}^{2}}{2}-\frac{T^{2}}{2}\right)+r\left(t_{1}^{2} T-\frac{2 t_{1}^{3}}{3}-\frac{T^{3}}{3}\right)\right]$

Thus, the total cost of the system per unit time is given by $\phi\left(t_{1}, T\right)=\frac{1}{T}\left[A_{0}+h_{0} q_{h c}+C_{0} q_{d c}+S_{0} q_{s c}\right]$
With the boundary conditions $q(0)=Q_{0}$ and $q\left(t_{1}\right)=0$

$$
\frac{d q(t)}{d t}=-R(t) \quad t_{1} \leq \mathrm{t} \leq \mathrm{T}
$$

Solution of equation (1) and (2) yields

$$
\begin{aligned}
& q(t)=a\left[\left(1-\theta_{0} t\right)\left(t_{1}-t\right)+\left(\frac{\theta_{0}}{2}-\frac{\theta_{0}{ }^{2} t}{2}\right)\left(t_{1}{ }^{2}-t^{2}\right)+\right. \\
& \left.\left(\frac{r}{2}-\frac{\theta_{0} r t}{2}\right)\left(t_{1}{ }^{2}-t^{2}\right)+\left(\frac{\theta_{0} r}{3}-\frac{\theta_{0}{ }^{2} r t}{3}\right)\left(t_{1}^{3}-t^{3}\right)\right]
\end{aligned}
$$

and

$q(t)=a\left[\left(t_{1}-t\right)+r\left(\frac{t_{1}^{2}}{2}-\frac{t^{2}}{2}\right)\right]$

By using $I\left(t_{1}\right)=0$, we have

$Q_{0}=a\left[t_{1}+\frac{\theta_{0} t_{1}{ }^{2}}{2}+\frac{r t_{1}{ }^{2}}{2}+\frac{\theta_{0} r t_{1}^{3}}{3}\right]$

Holding cost $\left(q_{h c}\right)$ in the cycle is given by

$$
\begin{aligned}
q_{h c}= & \int_{0}^{t_{1}} q(t)=a\left[\frac{3 t_{1}^{2}}{2}+\frac{2 \theta_{0} t_{1}^{3}}{3}+\frac{2 r t_{1}^{3}}{3}+\frac{5 \theta_{0} r t_{1}^{4}}{12}\right. \\
& \left.-\frac{\theta_{0} t_{1}{ }^{3}}{6}-\frac{\theta_{0}{ }^{2} t_{1}{ }^{4}}{8}-\frac{\theta_{0} r t_{1}{ }^{4}}{8}-\frac{\theta_{0}{ }^{2} r t_{1}{ }^{5}}{10}\right]
\end{aligned}
$$

$$
\phi\left(t_{1}, T\right)=\frac{1}{T}\left[\begin{array}{c}
A_{0}+h_{0} a\left(\begin{array}{c}
\frac{3 t_{1}^{2}}{2}+\frac{2 \theta_{0} t_{1}^{3}}{3}+\frac{2 r t_{1}^{3}}{3} \\
+\frac{5 \theta_{0} r t_{1}{ }^{3}}{12}-\frac{\theta_{0} t_{1}^{3}}{6}-\frac{\theta_{0}{ }^{2} t_{1}{ }^{4}}{8} \\
-\frac{\theta_{0} r t_{1}{ }^{4}}{8}-\frac{\theta_{0}{ }^{2} r t_{1}{ }^{5}}{10}
\end{array}\right) \\
+C_{0}\left(\frac{\theta_{0} t_{1}^{2}}{2}+\frac{\theta_{0} r t_{1}^{3}}{3}\right)-S_{0}\left(\begin{array}{l}
a\left(t_{1} T-\frac{t_{1}^{2}}{2}-\frac{T^{2}}{2}\right) \\
r\left(t_{1}^{2} T-\frac{2 t_{1}^{3}}{3}-\frac{T^{3}}{3}\right)
\end{array}\right)
\end{array}\right]
$$




\section{Fuzzy Model:}

Due to uncertainty in the environment, we assume some of the parameters of the inventory system like $a, h_{0}, S_{0}, r, \theta_{0}$ and $C_{0}$ may change within certain limits. Here we have considered $\tilde{a}, \tilde{h}_{0}, \tilde{S}_{0}, \tilde{r}, \tilde{\theta}_{0}$ and $\tilde{C}_{0}$ are pentagonal fuzzy number. The fuzzy total cost is given by

$\tilde{\phi}\left(t_{1}, T\right)=\frac{1}{T}\left[\begin{array}{c}\tilde{A}_{0}+\tilde{h}_{0} \tilde{a}\left(\begin{array}{c}\frac{3 t_{1}^{2}}{2}+\frac{2 \tilde{\theta}_{0} t_{1}^{3}}{3}+\frac{2 \tilde{r} t_{1}^{3}}{3}+ \\ \frac{5 \tilde{\theta}_{0} \tilde{r} t_{1}^{4}}{12}-\frac{\tilde{\theta}_{0} t_{1}^{3}}{6}-\frac{\tilde{\theta}_{0}{ }^{2} t_{1}{ }^{3}}{8} \\ -\frac{\tilde{\theta}_{0} \tilde{r} t_{1}^{4}}{8}-\frac{\tilde{\theta}_{0}^{2} \tilde{r}_{1}{ }^{5}}{10}\end{array}\right)+ \\ \tilde{C}_{0}\left(\frac{\tilde{\theta}_{0} t_{1}^{2}}{2}+\frac{\tilde{\theta}_{0} \tilde{r} t_{1}^{3}}{3}\right)-\tilde{S}_{0}\left(\begin{array}{l}\tilde{a}\left(t_{1} T-\frac{t_{1}^{2}}{2}-\frac{T^{2}}{2}\right)+ \\ \tilde{r}\left(t_{1}^{2} T-\frac{2 t_{1}^{3}}{3}-\frac{T^{3}}{3}\right)\end{array}\right)\end{array}\right]$
The total profit $\tilde{\phi}\left(t_{1}, T\right)$ has been defuzzified by employing signed distance method and graded mean representation method. When $\tilde{a}=\left(a_{1}, a_{2}, a_{3}, a_{4}, a_{5}\right)$ $\tilde{h}_{0}=\left(h_{01}, h_{02}, h_{03}, h_{04}, h_{05}\right), \tilde{S}_{0}=\left(S_{01}, S_{02}, S_{03}, S_{04}, S_{05}\right)$ $, \tilde{r}=\left(r_{1}, r_{2}, r_{3}, r_{4}, r_{5}\right), \tilde{\theta}_{0}=\left(\theta_{01}, \theta_{02}, \theta_{03}, \theta_{04}, \theta_{05}\right)$ and $\tilde{C}_{0}=\left(C_{01}, C_{02}, C_{03}, C_{04}, C_{05}\right)$ are considered as pentagonal fuzzy numbers, by using graded mean representation method for defuzzification, we have

$$
\phi_{G M}\left(t_{1}, T\right)=\frac{1}{12}\left[\begin{array}{l}
\phi_{G M_{1}}\left(t_{1}, T\right)+3 \phi_{G M_{2}}\left(t_{1}, T\right) \\
+4 \phi_{G M_{3}}\left(t_{1}, T\right)+3 \phi_{G M_{4}}\left(t_{1}, T\right)+\phi_{G M_{5}}\left(t_{1}, T\right)
\end{array}\right]
$$

Where,

$$
\begin{aligned}
& \phi_{G M_{1}}\left(t_{1}, T\right)=\frac{1}{T}\left[\begin{array}{l}
\left.A_{0}+h_{01} a_{1}\left(\frac{3 t_{1}{ }^{2}}{2}+\frac{2 \theta_{02} t_{1}^{3}}{3}+\frac{2 r_{2} t_{1}^{3}}{3}+\frac{5 \theta_{02} r_{2} t_{1}^{4}}{12}-\frac{\theta_{02} t_{1}{ }^{3}}{6}-\frac{\theta_{02}{ }^{2} t_{1}^{4}}{8}-\frac{\theta_{02} r_{2} t_{1}{ }^{4}}{8}-\frac{\theta_{02}{ }^{2} r_{2} t_{1}{ }^{5}}{10}\right)+\right] \\
C_{02}\left(\frac{\theta_{02} t_{1}^{2}}{2}+\frac{\theta_{02} r_{2} t_{1}^{3}}{3}\right)-S_{02}\left(a_{2}\left(t_{1} T-\frac{t_{1}^{2}}{2}-\frac{T^{2}}{2}\right)+r_{2}\left(t_{1}^{2} T-\frac{2 t_{1}^{3}}{3}-\frac{T^{3}}{3}\right)\right)
\end{array}\right] \\
& \phi_{G M_{2}}\left(t_{1}, T\right)=\frac{1}{T}\left[\begin{array}{l}
A_{0}+h_{02} a_{2}\left(\frac{3 t_{1}^{2}}{2}+\frac{2 \theta_{02} t_{1}^{3}}{3}+\frac{2 r_{2} t_{1}^{3}}{3}+\frac{5 \theta_{02} r_{2} t_{1}^{4}}{12}-\frac{\theta_{02} t_{1}^{3}}{6}-\frac{\theta_{02}{ }^{2} t_{1}{ }^{4}}{8}-\frac{\theta_{02} r_{2} t_{1}{ }^{4}}{8}-\frac{\theta_{02}{ }^{2} r_{2} t_{1}{ }^{5}}{10}\right) \\
C_{02}\left(\frac{\theta_{02} t_{1}{ }^{2}}{2}+\frac{\theta_{02} r_{2} t_{1}^{3}}{3}\right)-S_{02}\left(a_{2}\left(t_{1} T-\frac{t_{1}{ }^{2}}{2}-\frac{T^{2}}{2}\right)+r_{2}\left(t_{1}{ }^{2} T-\frac{2 t_{1}{ }^{3}}{3}-\frac{T^{3}}{3}\right)\right)
\end{array}\right] \\
& \phi_{G M_{3}}\left(t_{1}, T\right)=\frac{1}{T}\left[\begin{array}{l}
A_{0}+h_{03} a_{3}\left(\frac{3 t_{1}{ }^{2}}{2}+\frac{2 \theta_{03} t_{1}{ }^{3}}{3}+\frac{2 r_{3} t_{1}{ }^{3}}{3}+\frac{5 \theta_{03} r_{3} t_{1}{ }^{4}}{12}-\frac{\theta_{03} t_{1}{ }^{3}}{6}-\frac{\theta_{03}{ }^{2} t_{1}{ }^{4}}{8}-\frac{\theta_{03} r_{3} t_{1}{ }^{4}}{8}-\frac{\theta_{03}{ }^{2} r_{3} t_{1}{ }^{5}}{10}\right)+ \\
C_{03}\left(\frac{\theta_{3} t_{1}{ }^{2}}{2}+\frac{\theta_{3} r_{3} t_{1}{ }^{3}}{3}\right)-S_{03}\left(a_{3}\left(t_{1} T-\frac{t_{1}{ }^{2}}{2}-\frac{T^{2}}{2}\right)+r_{3}\left(t_{1}{ }^{2} T-\frac{2 t_{1}{ }^{3}}{3}-\frac{T^{3}}{3}\right)\right)
\end{array}\right]
\end{aligned}
$$


$\phi_{G M_{4}}\left(t_{1}, T\right)=\frac{1}{T}\left[\begin{array}{l}A_{0}+h_{04} a_{4}\left(\frac{3 t_{1}{ }^{2}}{2}+\frac{2 \theta_{04} t_{1}{ }^{3}}{3}+\frac{2 r_{4} t_{1}{ }^{3}}{3}+\frac{5 \theta_{04} r_{4} t_{1}{ }^{4}}{12}-\frac{\theta_{04} t_{1}{ }^{3}}{6}-\frac{\theta_{04}{ }^{2} t_{1}{ }^{4}}{8}-\frac{\theta_{04} r_{4} t_{1}{ }^{4}}{8}-\frac{\theta_{04}{ }^{2} r_{4} t_{1}{ }^{5}}{10}\right)+ \\ C_{04}\left(\frac{\theta_{04} t_{1}{ }^{2}}{2}+\frac{\theta_{04} r_{4} t_{1}{ }^{3}}{3}\right)-S_{04}\left(a_{4}\left(t_{1} T-\frac{t_{1}{ }^{2}}{2}-\frac{T^{2}}{2}\right)+r_{4}\left(t_{1}{ }^{2} T-\frac{2 t_{1}{ }^{3}}{3}-\frac{T^{3}}{3}\right)\right)\end{array}\right]$

$\phi_{G M_{5}}\left(t_{1}, T\right)=\frac{1}{T}\left[\begin{array}{l}A_{0}+h_{05} a_{5}\left(\frac{3 t_{1}{ }^{2}}{2}+\frac{2 \theta_{05} t_{1}{ }^{3}}{3}+\frac{2 r_{5} t_{1}{ }^{3}}{3}+\frac{5 \theta_{05} r_{5} t_{1}{ }^{4}}{12}-\frac{\theta_{05} t_{1}{ }^{3}}{6}-\frac{\theta_{05}{ }^{2} t_{1}{ }^{4}}{8}-\frac{\theta_{05} r_{5} t_{1}{ }^{4}}{8}-\frac{\theta_{05}{ }^{2} r_{5} t_{1}{ }^{5}}{10}\right)+ \\ C_{05}\left(\frac{\theta_{05} t_{1}^{2}}{2}+\frac{\theta_{05} r_{5} t_{1}{ }^{3}}{3}\right)-S_{05}\left(a_{5}\left(t_{1} T-\frac{t_{1}{ }^{2}}{2}-\frac{T^{2}}{2}\right)+r_{5}\left(t_{1}{ }^{2} T-\frac{2 t_{1}{ }^{3}}{3}-\frac{T^{3}}{3}\right)\right)\end{array}\right]$

To minimize total cost function per unit time $\phi_{d G}\left(t_{1}, T\right)$, the optimal value of $t_{1}$ and $T$ can be obtained by solving the following equations:

$$
\frac{d \phi_{G M}\left(t_{1}, T\right)}{d t_{1}}=0 \text { and } \frac{d \phi_{G M}\left(t_{1}, T\right)}{d T}=0
$$

Equation (12) is equivalent to

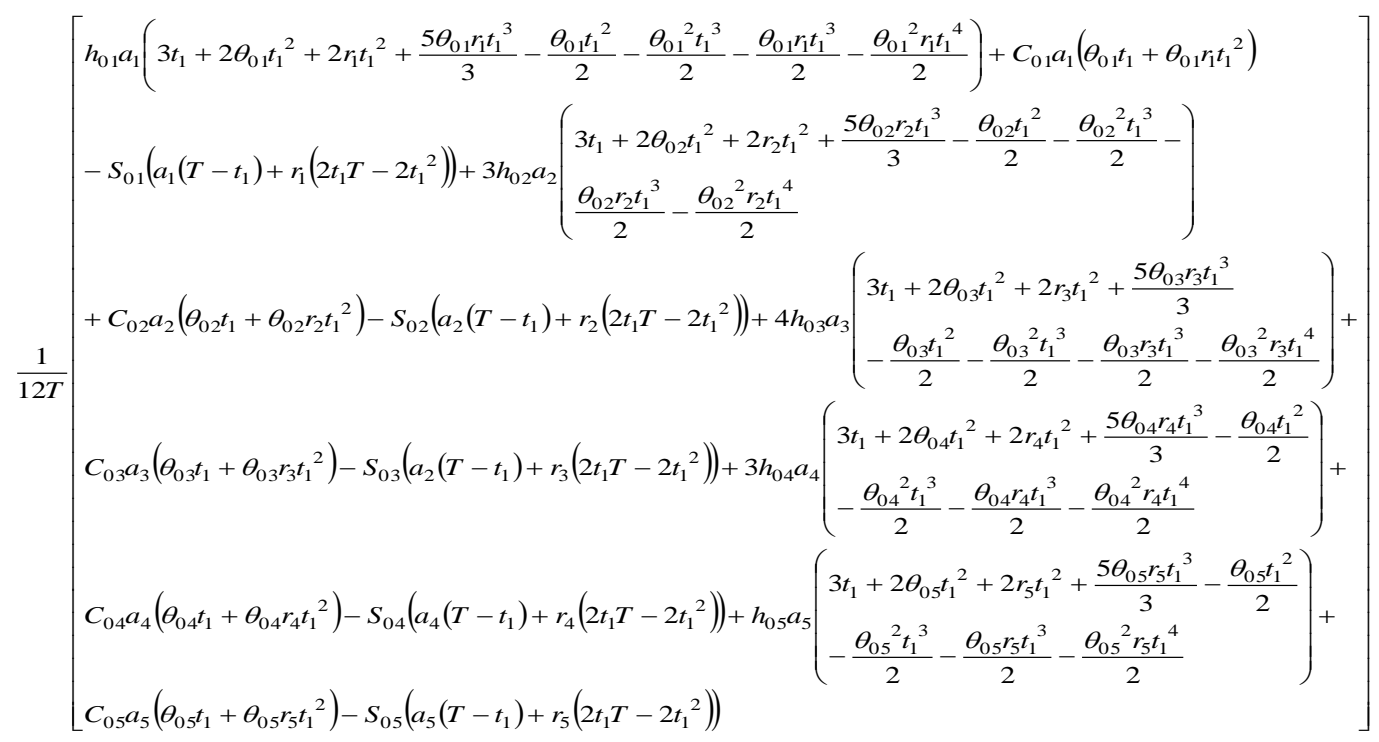




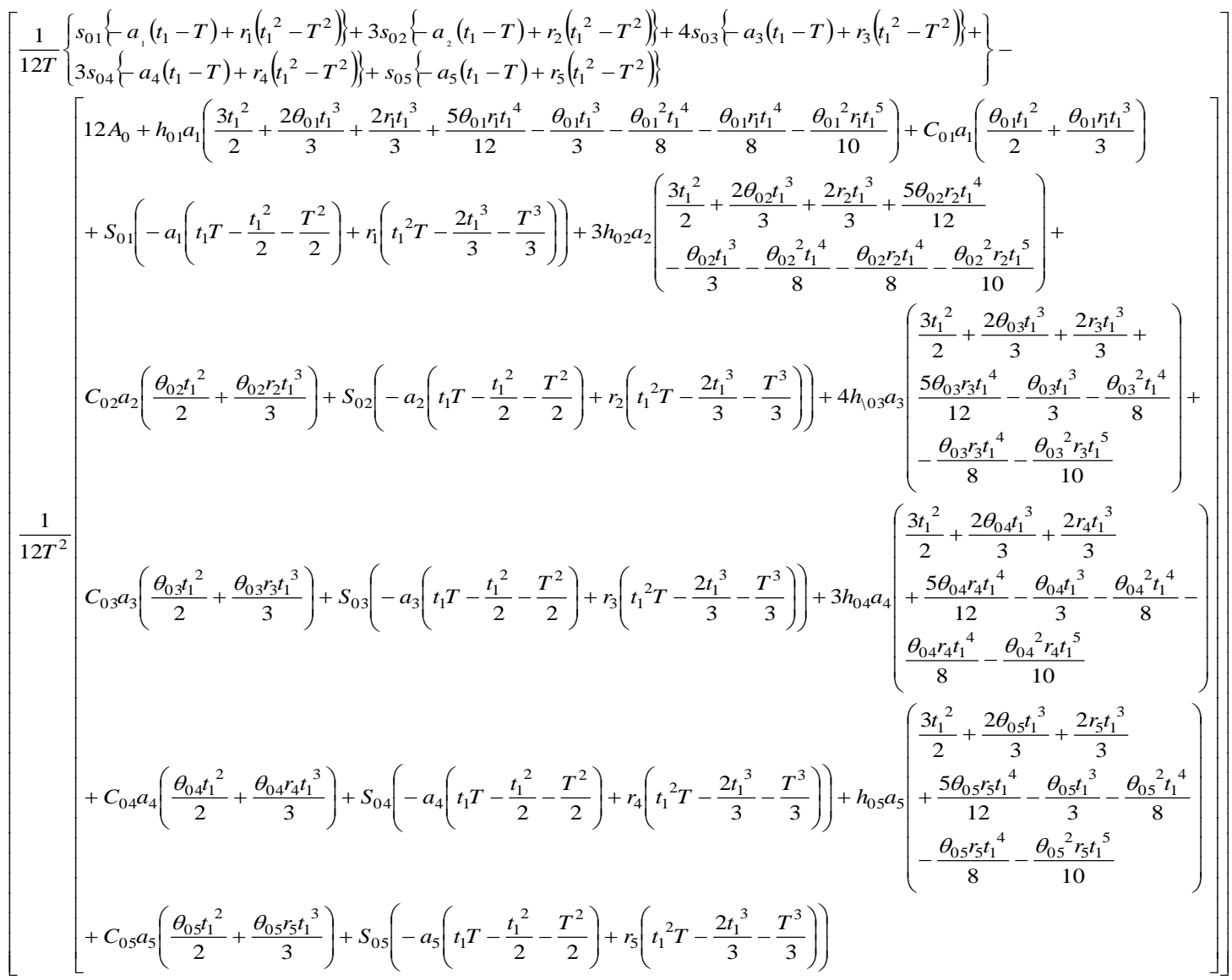

Further, for the total cost function $\phi_{d G}\left(t_{1}, T\right)$ to be convex, the following conditions must be satisfied

$\frac{d^{2} \phi_{G M}\left(t_{1}, T\right)}{d t_{1}^{2}}>0, \frac{d^{2} \phi_{G M}\left(t_{1}, T\right)}{d T^{2}}>0$,

and $\left(\frac{d^{2} \phi_{G M}\left(t_{1}, T\right)}{d t_{1}{ }^{2}}\right)\left(\frac{d^{2} \phi_{G M}\left(t_{1}, T\right)}{d T^{2}}\right)-\left(\frac{d^{2} \phi_{G M}\left(t_{1}, T\right)}{d t_{1} d T}\right)>0$

The second derivative of the total cost function $\phi_{d G}\left(t_{1}, T\right)$ is complicated and it is very difficult to prove its convexity mathematically. Thus, the convexity of total cost function has been established graphically (figure 1).

Using Signed Distance Method the, total cost is given by

$\phi_{S D}\left(t_{1}, T\right)=\frac{1}{8}\left[\phi_{S D_{1}}\left(t_{1}, T\right)+2 \phi_{S D_{2}}\left(t_{1}, T\right)+2 \phi_{S D_{3}}\left(t_{1}, T\right)+2 \phi_{S D_{4}}\left(t_{1}, T\right)+\phi_{S D_{5}}\left(t_{1}, T\right)\right]$

Where, 


$$
\begin{aligned}
& \phi_{S D_{1}}\left(t_{1}, T\right)=\frac{1}{T}\left[\begin{array}{l}
A_{0}+h_{01} a_{1}\left(\frac{3 t_{1}{ }^{2}}{2}+\frac{2 \theta_{01} t_{1}{ }^{3}}{3}+\frac{2 r_{1} t_{1}{ }^{3}}{3}+\frac{5 \theta_{01} r_{1} t_{1}{ }^{4}}{12}-\frac{\theta_{01} t_{1}{ }^{3}}{6}-\frac{\theta_{01}{ }^{2} t_{1}{ }^{4}}{8}-\frac{\theta_{01} r_{1} t_{1}{ }^{4}}{8}-\frac{\theta_{01}{ }^{2} r_{1} t_{1}{ }^{5}}{10}\right)+ \\
C_{01}\left(\frac{\theta_{01} t_{1}{ }^{2}}{2}+\frac{\theta_{01} r_{1} t_{1}{ }^{3}}{3}\right)-S_{01}\left(a_{1}\left(t_{1} T-\frac{t_{1}{ }^{2}}{2}-\frac{T^{2}}{2}\right)+r_{1}\left(t_{1}{ }^{2} T-\frac{2 t_{1}{ }^{3}}{3}-\frac{T^{3}}{3}\right)\right)
\end{array}\right] \\
& \phi_{S D_{2}}\left(t_{1}, T\right)=\frac{1}{T}\left[\begin{array}{l}
A_{0}+h_{02} a_{2}\left(\frac{3 t_{1}{ }^{2}}{2}+\frac{2 \theta_{02} t_{1}{ }^{3}}{3}+\frac{2 r_{2} t_{1}{ }^{3}}{3}+\frac{5 \theta_{02} r_{2} t_{1}{ }^{4}}{12}-\frac{\theta_{02} t_{1}{ }^{3}}{6}-\frac{\theta_{02}{ }^{2} t_{1}{ }^{4}}{8}-\frac{\theta_{02} r_{2} t_{1}{ }^{4}}{8}-\frac{\theta_{02}{ }^{2} r_{2} t_{1}{ }^{5}}{10}\right)+ \\
C_{02}\left(\frac{\theta_{02} t_{1}{ }^{2}}{2}+\frac{\theta_{02} r_{2} t_{1}{ }^{3}}{3}\right)-S_{02}\left(a_{2}\left(t_{1} T-\frac{t_{1}{ }^{2}}{2}-\frac{T^{2}}{2}\right)+r_{2}\left(t_{1}{ }^{2} T-\frac{2 t_{1}{ }^{3}}{3}-\frac{T^{3}}{3}\right)\right)
\end{array}\right] \\
& \phi_{S D_{3}}\left(t_{1}, T\right)=\frac{1}{T}\left[\begin{array}{l}
A_{0}+h_{03} a_{3}\left(\frac{3 t_{1}{ }^{2}}{2}+\frac{2 \theta_{03} t_{1}{ }^{3}}{3}+\frac{2 r_{3} t_{1}{ }^{3}}{3}+\frac{5 \theta_{03} r_{3} t_{1}{ }^{4}}{12}-\frac{\theta_{03} t_{1}{ }^{3}}{6}-\frac{\theta_{03}{ }^{2} t_{1}{ }^{4}}{8}-\frac{\theta_{03} r_{3} t_{1}{ }^{4}}{8}-\frac{\theta_{03}{ }^{2} r_{3} t_{1}{ }^{5}}{10}\right)+ \\
C_{03}\left(\frac{\theta_{03} t_{1}{ }^{2}}{2}+\frac{\theta_{03} r_{3} t_{1}{ }^{3}}{3}\right)-S_{03}\left(a_{3}\left(t_{1} T-\frac{t_{1}{ }^{2}}{2}-\frac{T^{2}}{2}\right)+r_{3}\left(t_{1}{ }^{2} T-\frac{2 t_{1}{ }^{3}}{3}-\frac{T^{3}}{3}\right)\right)
\end{array}\right] \\
& \phi_{S D_{4}}\left(t_{1}, T\right)=\frac{1}{T}\left[\begin{array}{l}
A_{0}+h_{04} a_{4}\left(\frac{3 t_{1}{ }^{2}}{2}+\frac{2 \theta_{04} t_{1}{ }^{3}}{3}+\frac{2 r_{4} t_{1}{ }^{3}}{3}+\frac{5 \theta_{04} r_{4} t_{1}{ }^{4}}{12}-\frac{\theta_{04} t_{1}{ }^{3}}{6}-\frac{\theta_{04}{ }^{2} t_{1}{ }^{4}}{8}-\frac{\theta_{04} r_{4} t_{1}{ }^{4}}{8}-\frac{\theta_{04}{ }^{2} r_{4} t_{1}{ }^{5}}{10}\right)+ \\
C_{04}\left(\frac{\theta_{04} t_{1}{ }^{2}}{2}+\frac{\theta_{04} r_{4} t_{1}{ }^{3}}{3}\right)-S_{04}\left(a_{4}\left(t_{1} T-\frac{t_{1}{ }^{2}}{2}-\frac{T^{2}}{2}\right)+r_{4}\left(t_{1}{ }^{2} T-\frac{2 t_{1}{ }^{3}}{3}-\frac{T^{3}}{3}\right)\right)
\end{array}\right] \\
& \phi_{S D_{5}}\left(t_{1}, T\right)=\frac{1}{T}\left[\begin{array}{l}
A_{0}+h_{05} a_{5}\left(\frac{3 t_{1}{ }^{2}}{2}+\frac{2 \theta_{05} t_{1}{ }^{3}}{3}+\frac{2 r_{5} t_{1}{ }^{3}}{3}+\frac{5 \theta_{05} r_{5} t_{1}{ }^{4}}{12}-\frac{\theta_{05} t_{1}{ }^{3}}{6}-\frac{\theta_{05}{ }^{2} t_{1}{ }^{4}}{8}-\frac{\theta_{05} r_{5} t_{1}{ }^{4}}{8}-\frac{\theta_{05}{ }^{2} r_{5} t_{1}{ }^{5}}{10}\right)+ \\
C_{05}\left(\frac{\theta_{05} t_{1}{ }^{2}}{2}+\frac{\theta_{05} r_{5} t_{1}{ }^{3}}{3}\right)-S_{05}\left(a_{5}\left(t_{1} T-\frac{t_{1}{ }^{2}}{2}-\frac{T^{2}}{2}\right)+r_{5}\left(t_{1}{ }^{2} T-\frac{2 t_{1}{ }^{3}}{3}-\frac{T^{3}}{3}\right)\right)
\end{array}\right]
\end{aligned}
$$

The total cost function $\phi_{S D}\left(t_{1}, T\right)$ has been minimized following the same process as has been stated above. To minimize total cost function per unit time $\phi_{S D}\left(t_{1}, T\right)$, the optimal value of $t_{1}$ and $T$ can be obtained by solving the following equations:

$$
\frac{d \phi_{S D}\left(t_{1}, T\right)}{d t_{1}}=0 \text { and } \frac{d \phi_{S D}\left(t_{1}, T\right)}{d T}=0
$$

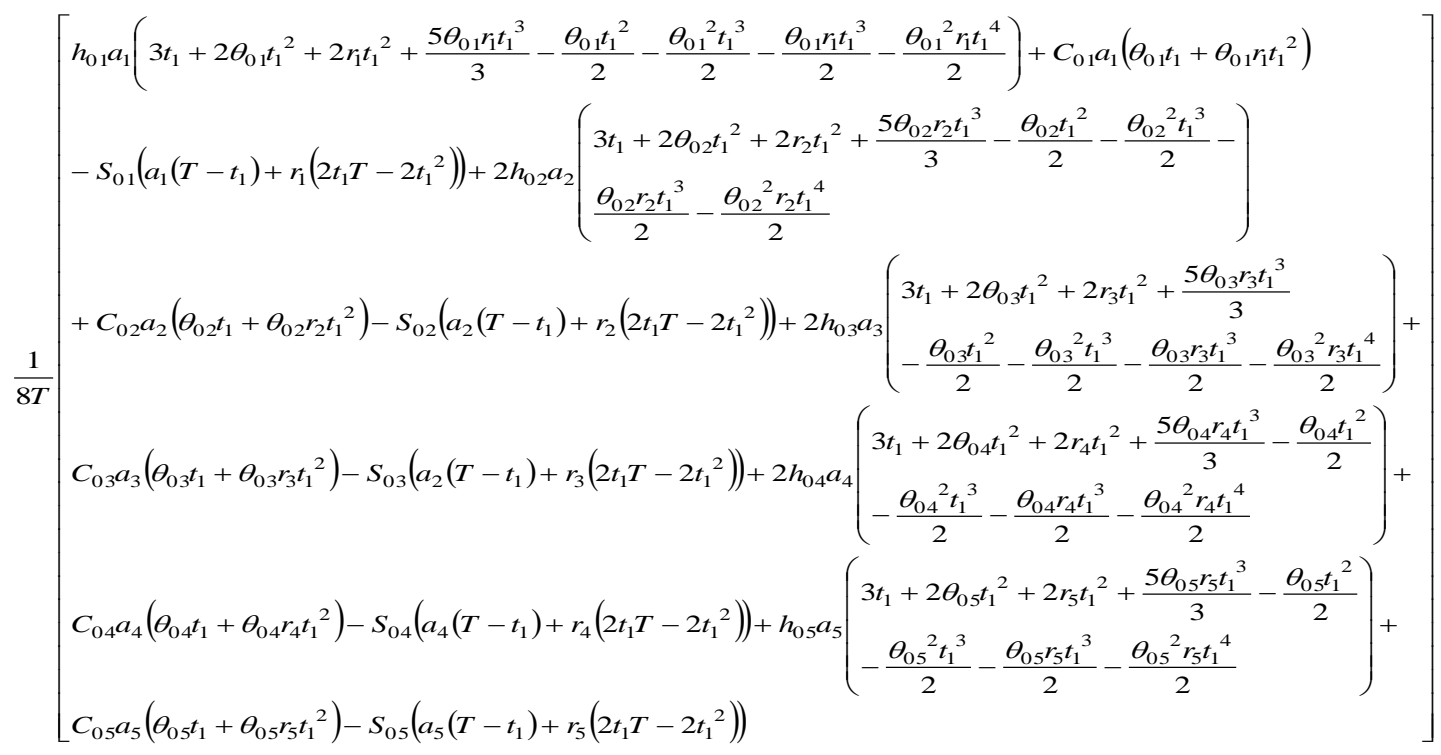




$$
\begin{aligned}
& {\left[\frac{1}{8 T}\left\{\begin{array}{l}
s_{01}\left\{-a_{1}\left(t_{1}-T\right)+r_{1}\left(t_{1}{ }^{2}-T^{2}\right)\right\}+3 s_{02}\left\{-a_{2}\left(t_{1}-T\right)+r_{2}\left(t_{1}{ }^{2}-T^{2}\right)\right\}+4 s_{03}\left\{\left(-a_{3}\left(t_{1}-T\right)+r_{3}\left(t_{1}{ }^{2}-T^{2}\right)\right\}+\right. \\
3 s_{04}\left\{-a_{4}\left(t_{1}-T\right)+r_{4}\left(t_{1}{ }^{2}-T^{2}\right)\right\}+s_{05}\left\{-a_{5}\left(t_{1}-T\right)+r_{5}\left(t_{1}{ }^{2}-T^{2}\right)\right\}
\end{array}\right\}-\right.} \\
& {\left[8 A+h_{01} a_{1}\left(\frac{3 t_{1}^{2}}{2}+\frac{2 \theta_{01} t_{1}^{3}}{3}+\frac{2 r_{1} t_{1}^{3}}{3}+\frac{5 \theta_{01} r_{1} t_{1}{ }^{4}}{12}-\frac{\theta_{01} t_{1}^{3}}{3}-\frac{\theta_{01}{ }^{2} t_{1}{ }^{4}}{8}-\frac{\theta_{01} r_{1} t_{1}{ }^{4}}{8}-\frac{\theta_{01}{ }^{2} r_{1} t_{1}{ }^{5}}{10}\right)+C_{01} a_{1}\left(\frac{\theta_{01} t_{1}{ }^{2}}{2}+\frac{\theta_{01} r_{1} t_{1}^{3}}{3}\right)\right.} \\
& +S_{01}\left(-a_{1}\left(t_{1} T-\frac{t_{1}{ }^{2}}{2}-\frac{T^{2}}{2}\right)+r_{1}\left(t_{1}^{2} T-\frac{2 t_{1}{ }^{3}}{3}-\frac{T^{3}}{3}\right)\right)+2 h_{02} a_{2}\left(\begin{array}{l}
\frac{3 t_{1}{ }^{2}}{2}+\frac{2 \theta_{02} t_{1}{ }^{3}}{3}+\frac{2 r_{2} t_{1}{ }^{3}}{3}+\frac{5 \theta_{02} r_{2} t_{1}{ }^{4}}{12} \\
-\frac{\theta_{02} t_{1}{ }^{3}}{3}-\frac{\theta_{02}{ }^{2} t_{1}{ }^{4}}{8}-\frac{\theta_{02} r_{2} t_{1}{ }^{4}}{8}-\frac{\theta_{02}{ }^{2} r_{2} t_{1}{ }^{5}}{10}
\end{array}\right)+ \\
& C_{02} a_{2}\left(\frac{\theta_{02} t_{1}^{2}}{2}+\frac{\theta_{02} r_{2} t_{1}^{3}}{3}\right)+S_{02}\left(-a_{2}\left(t_{1} T-\frac{t_{1}{ }^{2}}{2}-\frac{T^{2}}{2}\right)+r_{2}\left(t_{1}^{2} T-\frac{2 t_{1}{ }^{3}}{3}-\frac{T^{3}}{3}\right)\right)+2 h_{103} a_{3}\left(\begin{array}{l}
\frac{3 t_{1}{ }^{2}}{2}+\frac{2 \theta_{03} t_{1}{ }^{3}}{3}+\frac{2 r_{03} r_{3} t_{1}{ }^{3} t_{1}{ }^{4}}{3}-\frac{\theta_{03} t_{1}{ }^{3}}{3}-\frac{\theta_{03}{ }^{2} t_{1}{ }^{4}}{8} \\
-\frac{\theta_{03} r_{3} t_{1}{ }^{4}}{8}-\frac{\theta_{03}{ }^{2} r_{3} t_{1}{ }^{5}}{10}
\end{array}\right)+ \\
& \frac{1}{8 T^{2}} \\
& C_{03} a_{3}\left(\frac{\theta_{03} t_{1}{ }^{2}}{2}+\frac{\theta_{03} r_{3} t_{1}{ }^{2}}{3}\right)+S_{03}\left(-a_{3}\left(t_{1} T-\frac{t_{1}{ }^{2}}{2}-\frac{T^{2}}{2}\right)+r_{3}\left(t_{1}{ }^{2} T-\frac{2 t_{1}{ }^{3}}{3}-\frac{T^{3}}{3}\right)\right)+2 h_{04} a_{4}\left(\begin{array}{l}
\frac{3 t_{1}{ }^{2}}{2}+\frac{2 \theta_{4} t_{1}{ }^{3}}{3}+\frac{2 r_{4} t_{1}{ }^{3}}{3} \\
+\frac{5 \theta_{04} r_{4} t_{1}{ }^{4}}{12}-\frac{\theta_{04} t_{1}{ }^{3}}{3}-\frac{\theta_{04}{ }^{2} t_{1}{ }^{4}}{8}- \\
\frac{\theta_{04} r_{4} t_{1}{ }^{4}}{8}-\frac{\theta_{04}{ }^{2} r_{4} t_{1}{ }^{5}}{10}
\end{array}\right) \\
& \frac{\theta_{04} r_{4} t_{1}^{4}}{8}-\frac{\theta_{04}{ }^{2} r_{4} t_{1}^{5}}{10} \\
& +C_{04} a_{4}\left(\frac{\theta_{04} t_{1}^{2}}{2}+\frac{\theta_{04} r_{4} t_{1}^{3}}{3}\right)+S_{04}\left(-a_{4}\left(t_{1} T-\frac{t_{1}{ }^{2}}{2}-\frac{T^{2}}{2}\right)+r_{4}\left(t_{1}^{2} T-\frac{2 t_{1}^{3}}{3}-\frac{T^{3}}{3}\right)\right)+h_{05} a_{5}\left(\begin{array}{l}
\frac{3 t_{1}{ }^{2}}{2}+\frac{2 \theta_{05} t_{1}{ }^{3}}{3}+\frac{2 r_{5} t_{1}{ }^{3}}{3} \\
+\frac{5 \theta_{05} r_{5} t_{1}{ }^{4}}{12}-\frac{\theta_{05} t_{1}{ }^{3}}{3}-\frac{\theta_{05}{ }^{2} t_{1}{ }^{4}}{8} \\
-\frac{\theta_{05} r_{5} t_{1}{ }^{4}}{8}-\frac{\theta_{05}{ }^{2} r_{5} t_{1}{ }^{5}}{10}
\end{array}\right) \\
& +C_{05} a_{5}\left(\frac{\theta_{05} t_{1}^{2}}{2}+\frac{\theta_{05} r_{5} t_{1}^{3}}{3}\right)+S_{05}\left(-a_{5}\left(t_{1} T-\frac{t_{1}^{2}}{2}-\frac{T^{2}}{2}\right)+r_{5}\left(t_{1}^{2} T-\frac{2 t_{1}^{3}}{3}-\frac{T^{3}}{3}\right)\right)
\end{aligned}
$$

Further, for the total cost function $\phi_{S D}\left(t_{1}, T\right)$ to be convex, the following conditions must be satisfied

$$
\begin{aligned}
& \frac{d^{2} \phi_{S D}\left(t_{1}, T\right)}{d t_{1}{ }^{2}}>0, \frac{d^{2} \phi_{S D}\left(t_{1}, T\right)}{d T^{2}}>0 \\
& \left(\frac{d^{2} \phi_{S D}\left(t_{1}, T\right)}{d t_{1}{ }^{2}}\right)\left(\frac{d^{2} \phi_{S D}\left(t_{1}, T\right)}{d T^{2}}\right)-\left(\frac{d^{2} \phi_{S D}\left(t_{1}, T\right)}{d t_{1} d T}\right)>0
\end{aligned}
$$

The second derivative of the total cost function $\phi_{G M}\left(t_{1}, T\right)$ is complicated and it is very difficult to prove its convexity mathematically. Thus, the convexity of total cost function has been established graphically, (Figure 2). 


\section{NUMERICAL EXAMPLES}

For the illustration of proposed model we consider following inventory system in which values of different parameters in proper units.

\section{Crisp Model:}

$A_{0}=1500 /$ order, $\mathrm{h}_{0}=$ Rs.7/order, $a=100$ units /order,

$\theta_{0}=0.006 /$ year,$S_{0}=R s .4$ umits /year,

The

$C_{0}=R s .6$ /unit, $r=0.6$ units.

solution of the crisp model is

$\phi\left(t_{1}, T\right)=$ Rs. $385.8, t_{1}=0.7820$ year, $T=5.7310$

year.

\section{Fuzzy Model:}

$\tilde{h}_{0}=(3,5,7,9,11), \tilde{a}=(3,6,10,13,16)$,

$\tilde{\theta}_{0}=(0.002,0.004,0.006,0.008,0.01)$,

$\tilde{r}=(0.2,0.4,0.6,0.8,1), \tilde{C}_{0}=(2,4,6,8,10)$.

The Solution of the fuzzy model can be explored by the following two methods.

Table 1. Optimum values of $t_{1}, \mathrm{~T}$ and $\phi\left(t_{1}, T\right)$ when $\tilde{a}, \tilde{\mathrm{h}}_{0}, \tilde{\theta}_{0}, \tilde{\mathrm{r}}, \widetilde{\mathrm{C}}_{0}, \widetilde{\mathrm{S}}_{0}$ are pentagonal fuzzy numbers.

\begin{tabular}{|c|c|c|c|}
\hline $\begin{array}{c}\text { Methods } \\
\text { employed }\end{array}$ & $t_{1}$ & $\mathrm{~T}$ & $\phi\left(t_{1}, T\right)$ \\
\hline GMRM & 2.3202 & 21.0859 & 410.583 \\
\hline SDM & 2.2541 & 19.996 & 378.744 \\
\hline
\end{tabular}

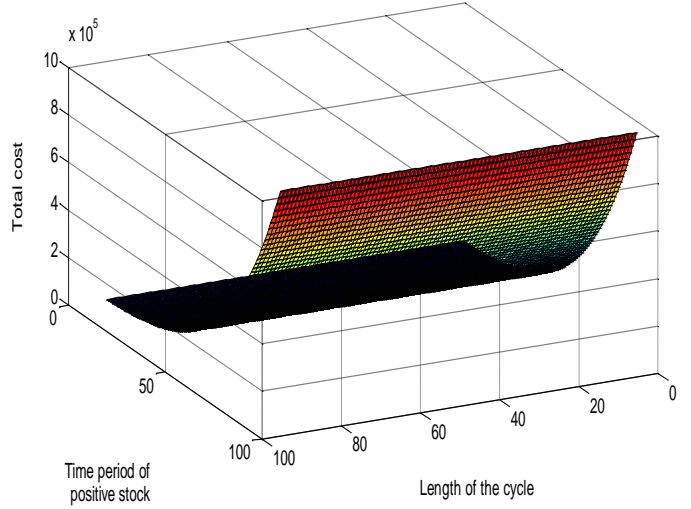

Figure 1. Total fuzzy cost $\phi_{G M}\left(t_{1}, T\right)$ vs. Time period of positive stock $\left(t_{1}\right)$ and Length of the cycle $(T)$
Table 2. Optimum values of $t_{1}$, T and $\phi\left(t_{1}, T\right)$ when

$\tilde{a}, \tilde{\theta}_{0}, \tilde{\mathrm{r}}, \widetilde{\mathrm{C}}_{0}, \widetilde{\mathrm{S}}_{0}$ are pentagonal fuzzy numbers.

\begin{tabular}{|c|c|c|c|}
\hline $\begin{array}{c}\text { Method } \\
\text { employed }\end{array}$ & $t_{1}$ & $\mathrm{~T}$ & $\phi\left(t_{1}, T\right)$ \\
\hline GMRM & 2.4959 & 20.9685 & 384.577 \\
\hline SDM & 2.4586 & 20.8406 & 369.883 \\
\hline
\end{tabular}

Table 3. Optimum values of $t_{1}$, T and $\phi\left(t_{1}, T\right)$ when $\tilde{a}, \widetilde{\theta}_{0}, \widetilde{\mathrm{r}}, \widetilde{\mathrm{C}}_{0}$ are pentagonal fuzzy numbers.

\begin{tabular}{|c|c|c|c|}
\hline $\begin{array}{c}\text { Method } \\
\text { employed }\end{array}$ & $t_{1}$ & $\mathrm{~T}$ & $\phi\left(t_{1}, T\right)$ \\
\hline GMRM & 2.2720 & 20.1058 & 337.881 \\
\hline SDM & 2.1975 & 18.9656 & 333.612 \\
\hline
\end{tabular}

Table 4. Optimum values of $t_{1}$, T and $\phi\left(t_{1}, T\right)$ when $\tilde{a}, \tilde{\theta}_{0}, \tilde{\mathrm{r}}$ are

\begin{tabular}{|c|c|c|c|}
\multicolumn{5}{c}{ pentagonal fuzzy numbers. } \\
\hline $\begin{array}{c}\text { Method } \\
\text { employed }\end{array}$ & $t_{1}$ & $\mathrm{~T}$ & $\phi\left(t_{1}, T\right)$ \\
\hline GMRM & 2.2727 & 20.1053 & 337.854 \\
\hline SDM & 2.1984 & 18.965 & 333.600 \\
\hline
\end{tabular}

Table 5. Optimum values of $t_{1}$, T and $\phi\left(t_{1}, T\right)$ when $\tilde{a}$ and $\tilde{\theta}_{0}$ are

\begin{tabular}{|c|c|c|c|}
\hline \multicolumn{4}{|c|}{ IZZY I } \\
\hline $\begin{array}{c}\text { Method } \\
\text { employed }\end{array}$ & $t_{1}$ & $\mathrm{~T}$ & $\phi\left(t_{1}, T\right)$ \\
\hline GMRM & 2.0563 & 17.8861 & 299.86 \\
\hline SDM & 2.0237 & 17.5304 & 298.545 \\
\hline
\end{tabular}

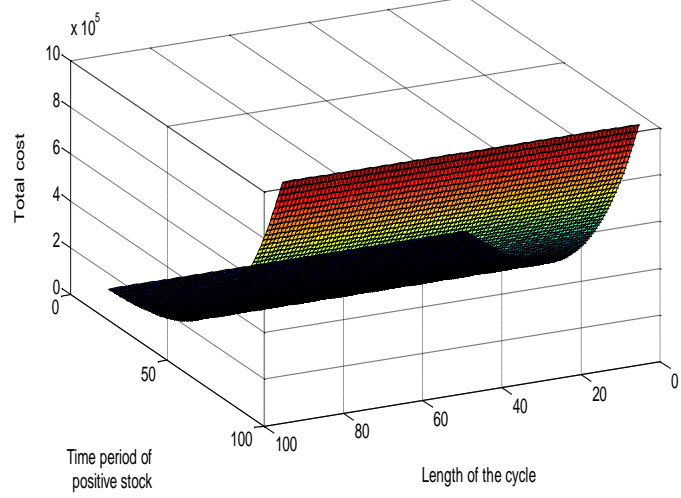

Figure 2. Total fuzzy Cost $\phi_{S D}\left(t_{1}, T\right)$ vs. Time period of positive stock $\left(t_{1}\right)$ and Length of the cycle $(T)$ 


\section{Sensitivity Analysis:}

A sensitivity analysis is performed to examine the impact and effect of changes in fuzzy parameter $\tilde{r}, \tilde{\theta}$ and $\tilde{\mathrm{h}}$ on the optimal solution by taking the defuzzified values of these parameters. The results are shown in below tables.

\section{By using Graded Mean Representation Method:}

Table 6. Sensitivity Analysis of reliability ( $r$ ) with time period of positive stock, length of the cycle and total cost

\begin{tabular}{|c|c|c|c|}
\hline$r$ & $t_{1}$ & $T$ & $\phi_{G M}\left(t_{1}, T\right)$ \\
\hline 0.2 & 6.0597 & 54.7609 & 1017.71 \\
\hline 0.4 & 3.1145 & 28.0512 & 520.718 \\
\hline 0.6 & 2.1424 & 19.3751 & 359.674 \\
\hline 0.8 & 1.6637 & 15.1476 & 281.307 \\
\hline 1 & 1.3801 & 12.664 & 235.312 \\
\hline
\end{tabular}

Table 7. Sensitivity Analysis of deterioration rate $\left(\theta_{0}\right)$ with time period of positive stock, length of the cycle and total cost

\begin{tabular}{|c|c|c|c|}
\hline$\theta_{0}$ & $t_{1}$ & $T$ & $\phi_{G M}\left(t_{1}, T\right)$ \\
\hline 0.002 & 2.3334 & 21.0791 & 391.131 \\
\hline 0.004 & 2.3283 & 21.0817 & 391.356 \\
\hline 0.006 & 2.3233 & 21.0843 & 391.578 \\
\hline 0.008 & 2.3183 & 21.0869 & 391.796 \\
\hline 0.01 & 2.3133 & 21.0894 & 392.01 \\
\hline
\end{tabular}

Table 8 . Sensitivity Analysis of holding cost $\left(h_{0}\right)$ with time period of positive stock, length of the cycle and total cost

\begin{tabular}{|c|c|c|c|}
\hline$h_{0}$ & $t_{1}$ & $T$ & $\phi_{G M}\left(t_{1}, T\right)$ \\
\hline 2 & 5.2957 & 19.5849 & 283.51 \\
\hline 3 & 4.2181 & 20.0062 & 319.17 \\
\hline 4 & 3.5567 & 20.3341 & 343.17 \\
\hline 5 & 3.0994 & 20.5915 & 360.644 \\
\hline 6 & 2.7600 & 20.7984 & 373.993 \\
\hline
\end{tabular}

\section{By using Signed Distance Method:}

Table 9. Sensitivity Analysis of reliability with time period of positive stock, length of the cycle and total cost

\begin{tabular}{|c|c|c|c|}
\hline$r$ & $t_{1}$ & $T$ & $\phi_{S D}\left(t_{1}, T\right)$ \\
\hline 0.2 & 5.761 & 51.7766 & 961.15 \\
\hline 0.4 & 2.9558 & 26.4661 & 490.764 \\
\hline 0.6 & 2.0291 & 18.2336 & 338.117 \\
\hline 0.8 & 1.5263 & 14.2191 & 263.771 \\
\hline 1 & 1.3622 & 11.8608 & 220.290 \\
\hline
\end{tabular}

Table 10. Sensitivity Analysis of deterioration rate with time period of positive stock, length of the cycle and total cost

\begin{tabular}{|c|c|c|c|}
\hline$\theta_{0}$ & $t_{1}$ & $T$ & $\phi_{S D}\left(t_{1}, T\right)$ \\
\hline 0.002 & 2.3515 & 20.9076 & 387.955 \\
\hline 0.004 & 2.3463 & 20.9106 & 388.188 \\
\hline 0.006 & 2.3413 & 20.9135 & 388.417 \\
\hline 0.008 & 2.3362 & 20.9164 & 388.343 \\
\hline 0.01 & 2.3313 & 20.9193 & 388.865 \\
\hline
\end{tabular}

Table 11. Sensitivity Analysis of holding cost with time period of positive stock, length of the cycle and total cost

\begin{tabular}{|c|c|c|c|}
\hline$h_{0}$ & $t_{1}$ & $T$ & $\phi_{S D}\left(t_{1}, T\right)$ \\
\hline 2 & 5.3432 & 19.2274 & 281.722 \\
\hline 3 & 4.2803 & 19.7206 & 319.093 \\
\hline 4 & 3.6200 & 20.0854 & 344.25 \\
\hline 5 & 3.1603 & 20.3649 & 362.506 \\
\hline 6 & 2.8176 & 20.5864 & 376.452 \\
\hline
\end{tabular}

Behavior of reliability, deterioration rate and holding cost in both the methods has been presented in the following figures.

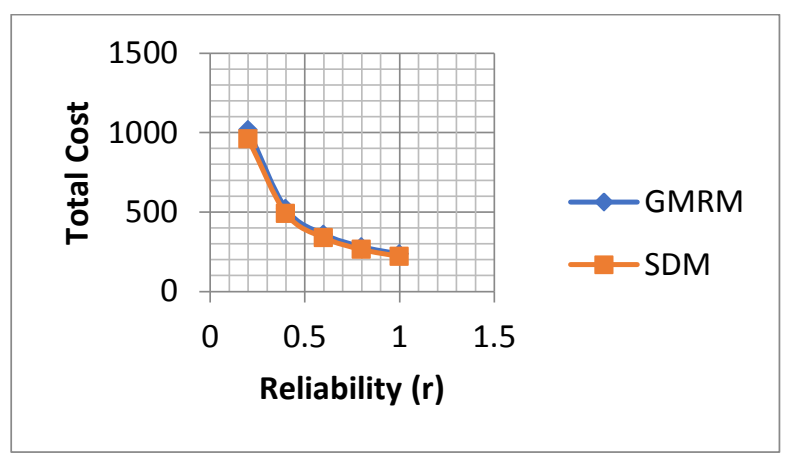

Figure 3. Behavior of reliability in GMRM and SDM

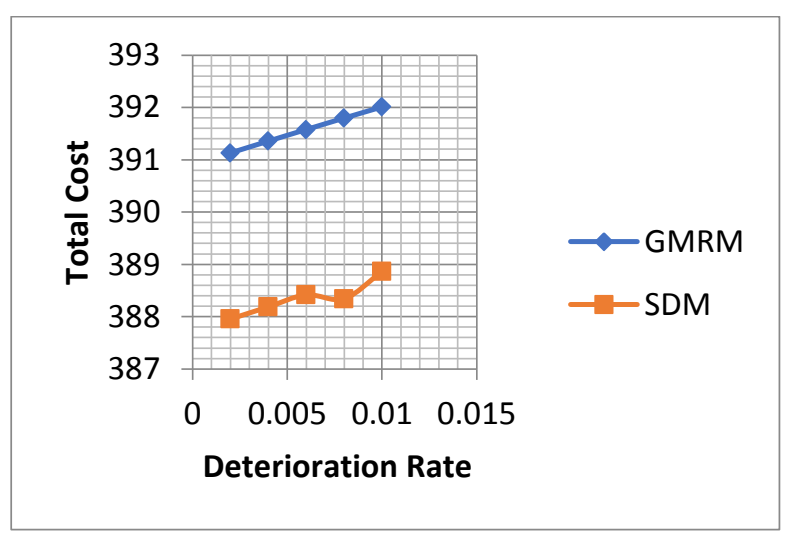

Figure 4. Behavior of deterioration rate in GMRM and SDM 


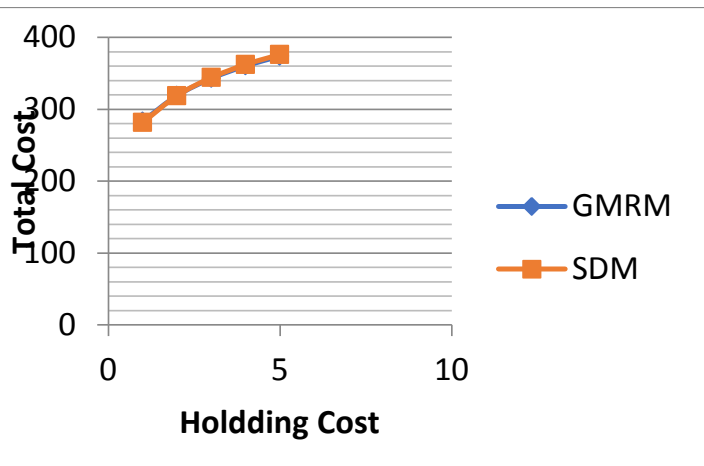

Figure 5. Behavior of holding cost in GMRM and SDM

\section{RESULT AND DISCUSSION}

- It is indicated in Table 6 and Table 9 that as the value of $r$ increases, total cost, total cycle length and time period of positive stock increase substantially.

- It is indicated in Table 7 and Table 10 that as the value of $\theta_{0}$ increases, total cost and total cycle length increase and time period of positive stock decreases gradually.

- It is indicated in Table 8 and Table 11 that as the value of $h_{0}$ increases, total cost and total cycle length increase and time period of positive stock decreases drastically.

\section{CONCLUSION}

In this paper, we have used signed distance method and graded mean representation method for defuzzying holding cost, setup cost, reliability, deterioration rate and shortage cost. These costs are taken as pentagonal fuzzy number. Demand is assumed to be an increasing function of time and reliability. It is seen from our analysis that increase reliability of the item gives rise to decrease cost as it is associated with quality assurances. So, the management has to fix up the reliability at certain level depending on the cost of investment.

The proposed model can be extended by introducing trade credit, weibull deterioration and partial backlogging.

\section{Acknowledgement:}

The first author would like to acknowledge the DST Inspire Fellowship for providing DST Inspire Fellowship vide letter no DST/Inspire Fellowship/150161.

\section{References}

[1] D. Dutta and P. Kumar, "Fuzzy Inventory Model Without Shortage using Trapezoidal Fuzzy Number with Sensitivity Analysis", IOSR Journal of Mathematics, Vol.4, No. 3, pp.3237, 2012.

[2] D. Dutta and P. Kumar, "Optimal Ordering Policy for an Inventory Model for Deteriorating Items without Shortages by Considering Fuzziness in Demand Rate, Ordering Cost and Holding Cost", International Journal of Advanced Innovation and Research, Vol. 2, No. 3, pp.320-325, 2012.

[3] P. K. Tripathy, P. Tripathy, M. Pattnaik, "A fuzzy EOQ model with reliability and demand depended unit cost", International Journal of Contemporary Mathematics Science, Vol.6, No. 30, pp.1467-1482, 2011.

[4] H. J. Zimmermann, "Fuzzy Set Theory-and its Applications", Springer Science + Business Media, LLC, $4^{\text {th }}$ edition.

[5] P. K. Tripathy, M. Pattnaik, "Optimal Disposal Mechanism with Fuzzy System Cost under Flexibility and Reliability Criteria in Non-random Optimization Environment", Applied Mathematical Sciences, Vol.3, No.37, pp. 1823-1847, 2009.

[6] P. K. Tripathy, N. P. Behera, "Fuzzy EOQ Model for TimeDeteriorating Items Using Penalty Cost", American Journal of Operational Research, Vol.6, No.1, pp.1-8, 2016.

[7] S. R. Singh, H. Rathor, "An Inventory Model for Deteriorating Items with Reliability Consideration and Trade Credit", Pakistan Journal of Statistics and Operations Research, Vol. X, No. 3, pp.349-360, 2014.

[8] C.K. Jaggi, S. Pareek, A. Sharma, Nidhi, "Fuzzy Inventory Model for Deteriorating Items with Time-Varying Demand and Shortages", American Journal of Operational Research, Vol.2, No.6, pp.81-92, 2012.

\section{AUTHORS PROFILE:}

Nalini Prava Behera, INSPIRE Fellow, is now pursuing her Ph.D. in P.G. Department of Statistics, Utkal University. Her field of research is Operation Research.

P.K. Tripathy is the senior most Professor of the P.G. Department of Statistics, Utkal University and Director of Centre for IT Education, Utkal University. He has 29 years of teaching experience at P.G. level and 27 years of research experience. He pursued MSc. (Statistics), Utkal University, CPS (Population Studies), IIPS, Mumbai and Ph.D. (Statistics)

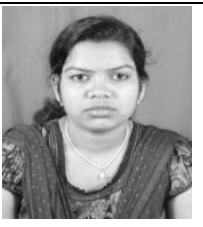
from Utkal University. His field of research is Operations Research. He has published 70 research papers in international/national journals. He has produced 12 Doctorates and 5 are to be awarded.

Note: Utkal University is an A+ grade University as per NAAC and tier 1 University by the UGC. It is the oldest university of the state and the mother University of the State. 